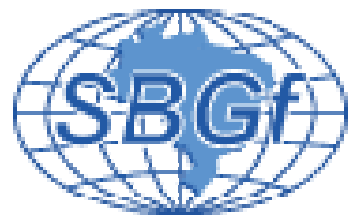

\title{
O EMPREGO DAS ONDAS DE RAYLEIGH PARA ESTIMATIVAS IN SITU DAS VELOCIDADES DAS ONDAS S DE SOLOS NÃO SATURADOS EM ÁREA SUJEITA A MOVIMENTOS DE MASSA
}

Sergio Bezerra Lima Júnior, Programa de pós-graduação IAG/USP

Renato Luiz Prado, IAG/USP

Rodolfo Moreda Mendes, IG/SMA

Copyright 2004, SBGf - Sociedade Brasileira de Geofísica

Este texto foi preparado para a apresentação no I Simpósio de Geofísica da Sociedade Brasileira de Geofísica, São Paulo, 26-28 de setembro de 2004. Seu conteúdo foi revisado pela Comissão Tecno-científica do I SR-SBGf mas não necessariamente representa a opinião da SBGf ou de seus associados. E proibida reproducão total ou parcial d autorização da SBGf.

\section{Resumo}

Este trabalho apresenta resultados preliminares de ensaios MASW (multi-channel analysis of surface waves) realizados em área de encosta sujeita a movimentos de massa. A pesquisa tem como objetivo a análise comparativa dos resultados obtidos em ensaios realizados em períodos distintos de pluviosidade numa área de solos não saturados, na região do município de Ubatuba, São Paulo. São apresentados os resultados preliminares das inversões realizadas a partir dos dados obtidos no período de menor pluviosidade com emprego de geofones de $4,5 \mathrm{~Hz}$ e afastamentos mínimos de 5 e 10 metros. Buscou-se analisar o quanto o aumento do afastamento afeta o resultado da inversão principalmente no que diz respeito ao detalhamento dos horizontes mais superficiais.Os modelos finais ajustados para os diferentes afastamentos mínimos não foram muito distintos em termos das profundidades ajustadas

\section{Introdução}

As relações existentes entre as propriedades elásticas dos materiais geológicos e as ondas sísmicas têm grande importância para várias áreas de aplicação da engenharia civil e geologia ambiental.

Além da obtenção dos chamados módulos elásticos dinâmicos a partir dos valores das velocidades das ondas $\mathrm{P}, \mathrm{S}$ e densidade dos materiais, há diversos estudos apresentando correlações empíricas entre os valores das velocidades sísmicas e parâmetros geotécnicos como número de golpes do ensaio SPT (Giacheti, 1991; Prado, 1994), grau de fraturamento (Turk \& Dearman, 1987), ou fator de qualidade do maciço (Barton et al., 1974), entre outros.

Em se tratando de materiais superficiais, o estudo da onda S é o que tem apresentado maior interesse para a mecânica de solos e geotecnia. A importância vem do fato da velocidade de propagação da onda S, Vs, estar diretamente relacionada à resistência do esqueleto estrutural dos solos através do qual a onda se propaga.
O conhecimento do módulo de cisalhamento dinâmico, G (equação 1) a partir dos valores de Vs, e das densidades dos materiais, $\rho$, é um dos principais parâmetros utilizados na previsão do comportamento tensãodeformação dos solos sob solicitação dinâmica de baixa amplitude.

$$
G=\rho V_{S}^{2} \text { (eq.1) }
$$

Neste trabalho busca-se a inversão da curva das velocidades de fase da onda Rayleigh para a obtenção do perfil de velocidade das ondas S, mas a partir de uma aquisição multicanal, assim como é executada na sísmica de reflexão convencional. Trata-se da técnica MASW (Park et al., 1999; Xia et al., 1999), de emprego ainda inédito no Brasil.

Apresenta-se neste trabalho uma discussão inicial dos primeiros resultados obtidos de ensaios realizados numa área de encosta com solos não saturados, na região de Ubatuba, local de ocorrência de numerosos movimentos de massa no Estado de São Paulo.

A pesquisa prevê também a análise comparativa dos resultados obtidos em ensaios realizados em dois períodos distintos de pluviosidade.

A razão deste procedimento é que existe uma clara associação dos escorregamentos da região da Serra do Mar com os períodos de intensificação do regime de chuvas. Nesse processo sabe-se do importante papel exercido pela sucção matricial. A baixos valores do teor de umidade têm-se altos valores de sucção matricial, gerando uma coesão adicional e conseqüente aumento da resistência ao cisalhamento.

A motivação desta pesquisa é verificar se o monitoramento intermitente do comportamento das ondas Rayleigh, e a conseqüente obtenção do perfil de velocidade das ondas $\mathrm{S}$ gera subsídio importante para a avaliação da estabilidade das encostas.

Porém o primeiro desafio desta pesquisa é resolver aspectos relacionados à aquisição e inversão dos dados que gerem modelos confiáveis e precisos o suficiente para que permitam a observação das eventuais alterações sazonais dos valores de Vs.

\section{Metodologia}

A aquisição foi realizada usando três grupos de 24 geofones de freqüências naturais de $4,5 \mathrm{~Hz}, 14 \mathrm{~Hz}$ e 28 $\mathrm{Hz}$ e uma fonte impulsiva do tipo martelo. Cada grupo de geofones constituiu um arranjo linear e os três arranjos foram posicionados paralelamente entre si a uma 
distância de 1 metro. O espaçamento entre geofones foi de 1 metro. Foram também testados diferentes afastamentos mínimos para a geração da onda, ou seja, desde 1 metro até 15 metros. O objetivo do emprego de diferentes configurações de aquisição foi o de analisar e estabelecer comparações entre seus conteúdos em relação ao registro das ondas de Rayleigh. Idealmente busca-se o registro do modo fundamental das ondas de Rayleigh numa larga banda de freqüências que permita 0 mapeamento de detalhe das camadas mais superficiais, assim como uma boa profundidade de investigação.

O processamento consiste, essencialmente, em obter a curva de dispersão a partir do sismograma analisado no domínio frequência-número de onda (f-k). O sismograma tempo-distância é transformado para o domínio f-k através da aplicação sucessiva de duas transformadas de Fourier. O espectro $\mathrm{f}-\mathrm{k}$ mostra as propriedades dispersivas das ondas de Rayleigh, permitindo restituir as diferentes velocidades de fase através da análise dos máximos de energia para cada freqüência. A partir desta análise é gerada a curva de dispersão, isto é, as diferentes velocidades de fase para cada freqüência.

A natureza multi-modal das ondas de Rayleigh é freqüentemente observada no gráfico de dispersão, através dos máximos energéticos presentes. A pesquisa de outros máximos relativos permite desenhar as curvas de dispersão para os diferentes modos de propagação. Uma das principais dificuldades no processamento das ondas superficiais está na separação entre os diversos modos de propagação, que nem sempre estão bem diferenciados.

A curva de dispersão é posteriormente utilizada para inferir o perfil de variação das velocidades das ondas cisalhantes e conseqüentemente do módulo de rigidez a partir de um processo de modelagem inversa. Este processo é iterativo e requer inicialmente estimativas das espessuras das camadas e seus respectivos valores do coeficiente de Poisson e das velocidades das ondas $\mathrm{S}$. $\mathrm{Na}$ nossa pesquisa temos empregado 0 aplicativo Surfseis desenvolvido pelo Serviço Geológico de Kansas (Park et al., 1999).

\section{Resultados e discussões}

A área de estudo está localizada no município de Ubatuba, litoral Norte do estado de São Paulo (Figura 1).

Suas características geológico-geotécnicas mais relevantes incluem: i) média encosta com espessura de solo reduzida; ii) perfil geológico básico composto de horizonte superficial de origem residual sobreposto a solo saprolítico e/ou saprolito na transição para o maciço rochoso

A partir da análise granulométrica de amostras de sondagens a trado e mapeamento de campo foi feita uma classificação expedita dos horizontes de solo presentes na área. (Figura 2).

Assim, tem-se para o solo residual três horizontes distintos: o primeiro composto por argila pouco arenosa, amarela, com grânulos de quartzo intercalados e com a presença de material orgânico intercalado $(0-0,8 \mathrm{~m})$; o segundo composto por material argiloso pouco arenoso, minerais micáceos, com pequenos grãos de quartzo dispersos, sem estrutura da rocha mãe $(0,8-5 \mathrm{~m})$; o terceiro de material areno-argiloso a argilo-arenoso, de cor variegada e com a presença de estrutura reliquiar (5 $-12 \mathrm{~m})$.

Abaixo do solo residual tem-se a rocha sã alterada composta de granito-gnaisses.

A Figura 2 apresenta as variações granulométricas dos diferentes horizontes com a profundidade.

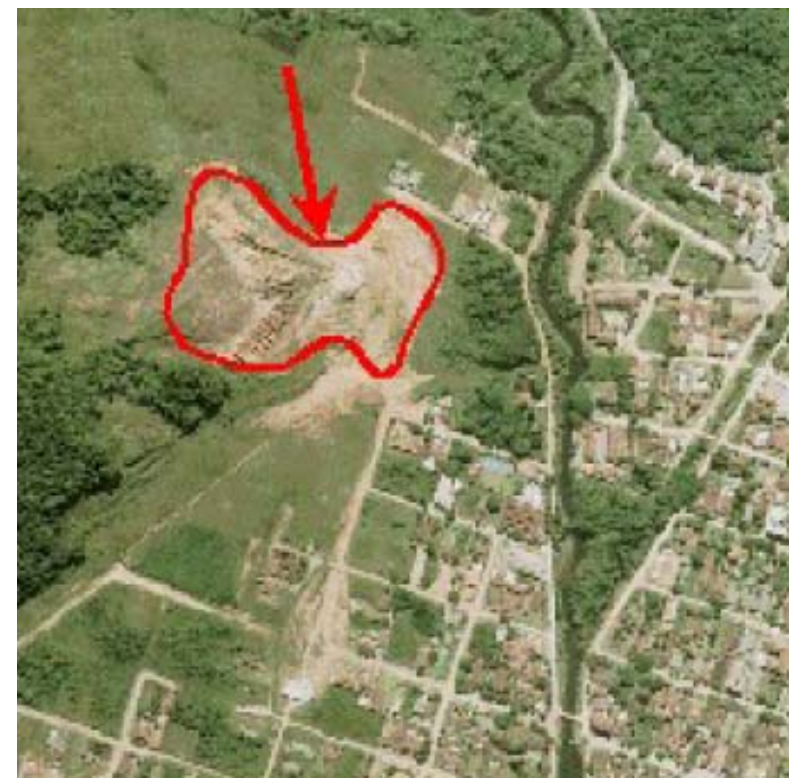

Figura 1 - Localização da área de estudo, na praia de Maranduba, Ubatuba-SP (Fonte: Ortofoto Digital de 2001 - Acervo IG/SMA)

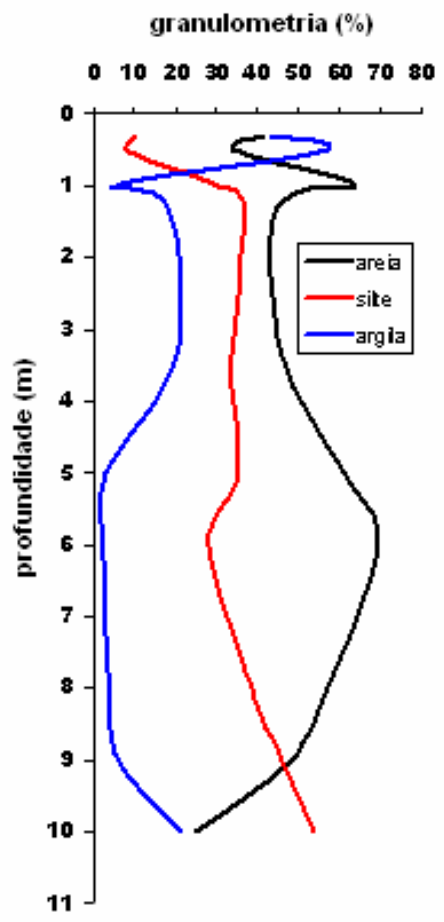

Figura 2 - Curvas das variações granulométricas das amostras coletadas no local dos ensaios geofísicos 
Os resultados preliminares das inversões aqui apresentados referem-se aos dados obtidos com os geofones de $4,5 \mathrm{~Hz}$ empregando os afastamentos mínimos de 5 e 10 metros.

Buscou-se analisar o quanto o aumento do afastamento afeta o resultado da inversão quanto ao detalhamento dos horizontes mais superficiais.

O modelo inicial para o processo de inversão baseou-se nas informações geológicas disponíveis e em dados de sísmica de refração realizados no local dos ensaios e é apresentado na Tabela 1.

Embora tenham sido empregados geofones de baixa freqüência $(4,5 \mathrm{~Hz})$ pode-se observar pelos espectros de amplitude (Figura 3) que a energia está concentrada numa faixa de freqüência superior a $15 \mathrm{~Hz}$, ou seja, aparentemente o meio não favorece a geração das baixas freqüências e a mudança de afastamento também não causou uma filtragem significativa das freqüências mais baixas (Figuras 4 e 5). Para a área em estudo talvez fosse mais adequado o uso de fontes de impacto de maior massa.

Os máximos energéticos observados para as baixas freqüências e altas velocidades de fases se relacionam às ondas de corpo.

Para a identificação inicial da curva de dispersão escolheu-se um ponto inicial de referência do gráfico de dispersão onde a acumulação de energia era alta e de características (velocidade de fase e freqüência) compatíveis às do modo fundamental das ondas de Rayleigh. Busca automática para a obtenção da curva de dispersão usando o aplicativo Surfeis muitas vezes resultou em curvas com quebras de coerência e ou inclusão de energias de modos superiores que tiveram que ser manualmente corrigidas.

A partir da curva final escolhida foi iniciado o processo de inversão para produzir o perfil Vs 1-D. O processo era finalizado sob critérios de valores mínimos de erro RMS adotados.

Os modelos finais ajustados empregando dados de afastamentos mínimos de 5 e 10 metros não foram muito distintos em termos das profundidades ajustadas, embora o melhor ajuste quanto aos valores de velocidades para os horizontes superficiais tenha sido obtido com os dados da aquisição feita com afastamento mínimo de 10 metros e para os horizontes mais profundos com os dados da aquisição com afastamento mínimo de 5 metros (Tabela 1).

O perfil final obtido revela um aumento crescente de Vs com a profundidade, desde valores próximos a $150 \mathrm{~m} / \mathrm{s}$ para os horizontes superficiais de solo residual de menor resistência e maior conteúdo em argila até valores de aproximadamente $1000 \mathrm{~m} / \mathrm{s}$ para o saprolito composto de granito-gnaisse bastante alterado.

$\mathrm{Na}$ continuidade da pesquisa serão interpretados todos os sismogramas (com diferentes afastamentos), inclusive os obtidos com os geofones de $14 \mathrm{~Hz}$ e $28 \mathrm{~Hz}$, empregando alguns filtros de freqüência tipo passabanda buscando analisar o quanto esses parâmetros (de aquisição e processamento) melhoram o resultado do gráfico de dispersão de energia.

A partir do melhor parâmetro encontrado serão refeitos os processos de inversão.
Tabela 1 - Dados do modelo inicial adotado para a inversão das curvas de dispersão e resultados dos processos de inversão para as curvas de dispersão obtidas (afastamentos mínimos de 5 e 10 metros)

\begin{tabular}{|c|c|c|c|}
\hline \multicolumn{4}{|c|}{ Modelo inicial } \\
\hline camada & $\begin{array}{l}\text { profundidade } \\
\text { (m) }\end{array}$ & $\begin{array}{c}\text { velocidade } \\
\text { onda S } \\
(\mathrm{m} / \mathrm{s})\end{array}$ & $\begin{array}{c}\text { coef. } \\
\text { Poisson }\end{array}$ \\
\hline 1 & 0,5 & 180 & 0.30 \\
\hline 2 & 4,76 & 350 & 0.30 \\
\hline 3 & 10,76 & 600 & 0.30 \\
\hline \multicolumn{2}{|c|}{ Semi espaço } & 1200 & 0.25 \\
\hline \multicolumn{4}{|c|}{ Afastamento mínimo de $5 \mathrm{~m}$} \\
\hline camada & $\begin{array}{l}\text { profundidade } \\
(\mathrm{m})\end{array}$ & $\begin{array}{c}\text { velocidade } \\
\text { onda S } \\
(\mathrm{m} / \mathrm{s})\end{array}$ & $\begin{array}{c}\text { coef. } \\
\text { Poisson }\end{array}$ \\
\hline 1 & 0,5 & 90 & 0.30 \\
\hline 2 & 4,76 & 229 & 0.30 \\
\hline 3 & 10,76 & 596 & 0.30 \\
\hline \multicolumn{2}{|c|}{ Semi espaço } & 1062 & 0.25 \\
\hline \multicolumn{4}{|c|}{ Afastamento mínimo de $10 \mathrm{~m}$} \\
\hline camada & $\begin{array}{l}\text { profundidade } \\
(\mathrm{m})\end{array}$ & $\begin{array}{c}\text { velocidade } \\
\text { onda S } \\
(\mathrm{m} / \mathrm{s})\end{array}$ & $\begin{array}{c}\text { coef. } \\
\text { Poisson }\end{array}$ \\
\hline 1 & 0,5 & 153 & 0,30 \\
\hline 2 & 4,76 & 333 & 0,30 \\
\hline 3 & 10,76 & 575 & 0,30 \\
\hline \multicolumn{2}{|c|}{ Semi espaço } & 785 & 0,25 \\
\hline
\end{tabular}

\section{Agradecimentos}

Os autores agradecem ao IAG/USP, IG/SMA, FAPESP, CAPES e ao Prof. Augustinho Rigoti pelos apoios dados para o desenvolvimento desta pesquisa.

\section{Referências}

Barton, N., Lien, R.; Lunde, J. 1974. Engineering classification of rock masses for the design of tunnel support. Rock Mechanics. 6: 4: 189-236.

Giacheti, H. L. Estudo experimental de parâmetros dinânicos de alguns solos tropicais do Estado de SãoPaulo. São Paulo, 1991, 232 p. Tese (Doutorado) Escola de Engenharia de São Carlos, Universidade de São Paulo.

Park, C. B., Miller, R. D., and Xia, J., 1999, Multi-channel analysis of surface waves: Geophysics, 64, 800-808.

Prado, R.L. O ensaio sísmico entre furos ("crosshole") no estudo de maciços terrosos e rochosos. São Paulo, 1994. 123 p. Dissertação (Mestrado em Geofísica) - Instituto Astronômico e Geofísico, Universidade de São Paulo.

Turk, N.; Dearman, W.R. Assessment of grounting efficiency in a rock mass in terms of seismic velocities. Bulletin of IAEG n.36, France, p.101-108, 1987.

Xia, J.; Xia, J.; Miller, R.D.; Park, C.B. 1999. Estimation of near-surface shear-wave velocity by inversion of Rayleigh waves. Geophysics, v.64, n.3. p.691-700. 

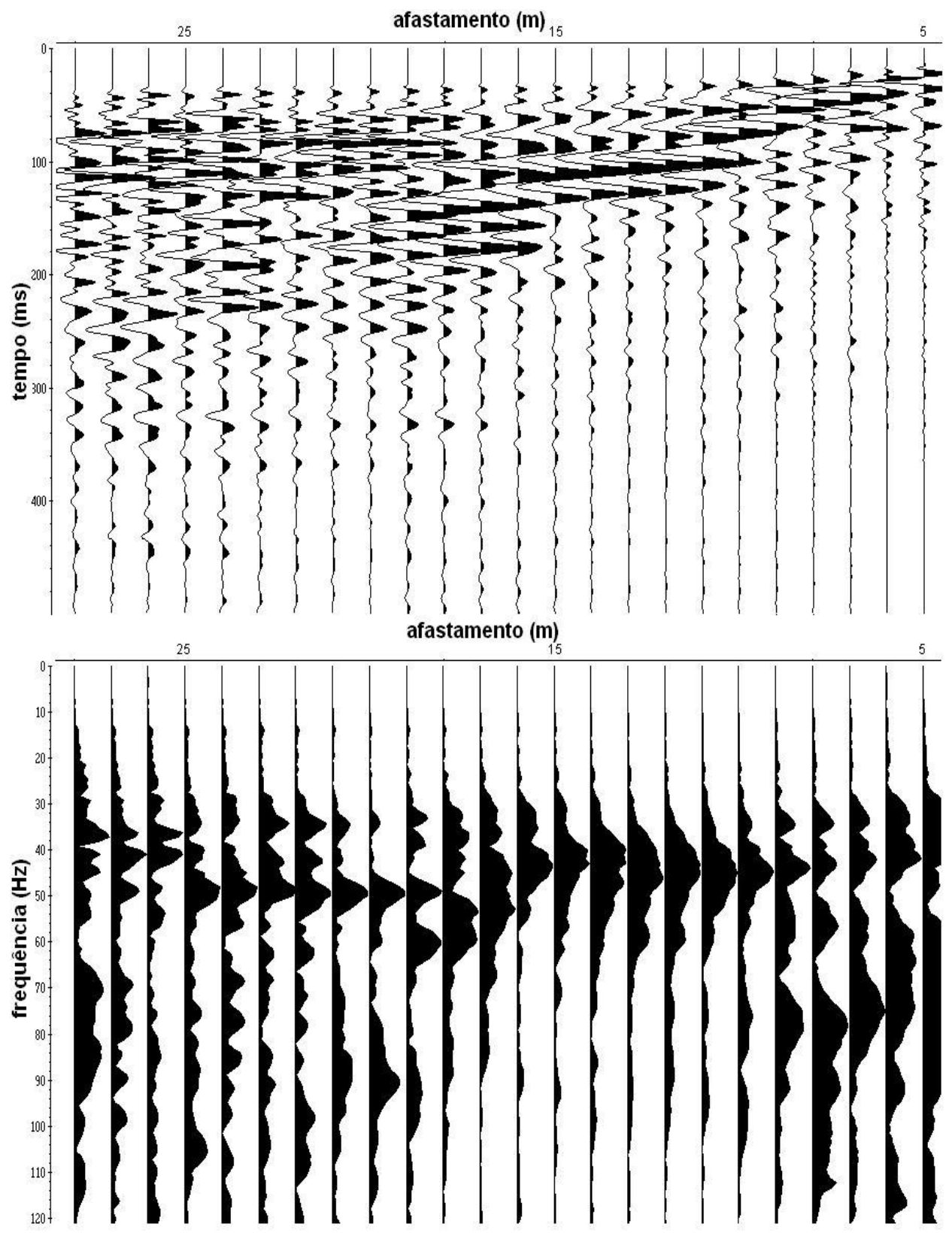

Figura 3 - Sismograma obtido com geofones de $4,5 \mathrm{~Hz}$ e afastamento mínimo de $5 \mathrm{~m}$ e espectros de amplitude de seus traços sísmicos. 

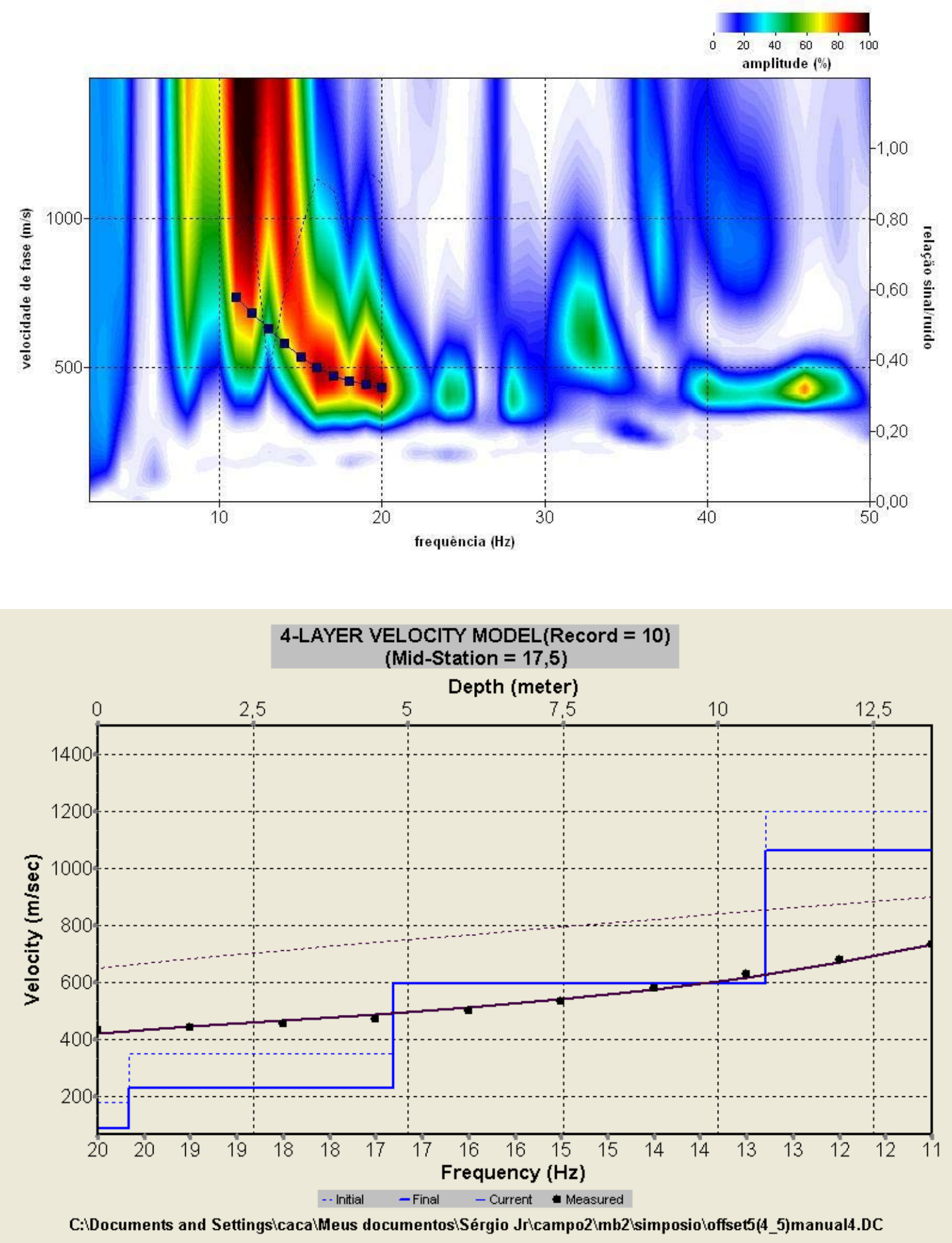

Figura 4 - Curva de dispersão e modelo final de inversão obtidos dos sismogramas adquiridos com geofones de 4,5 Hz e afastamento mínimo de 5 metros. 

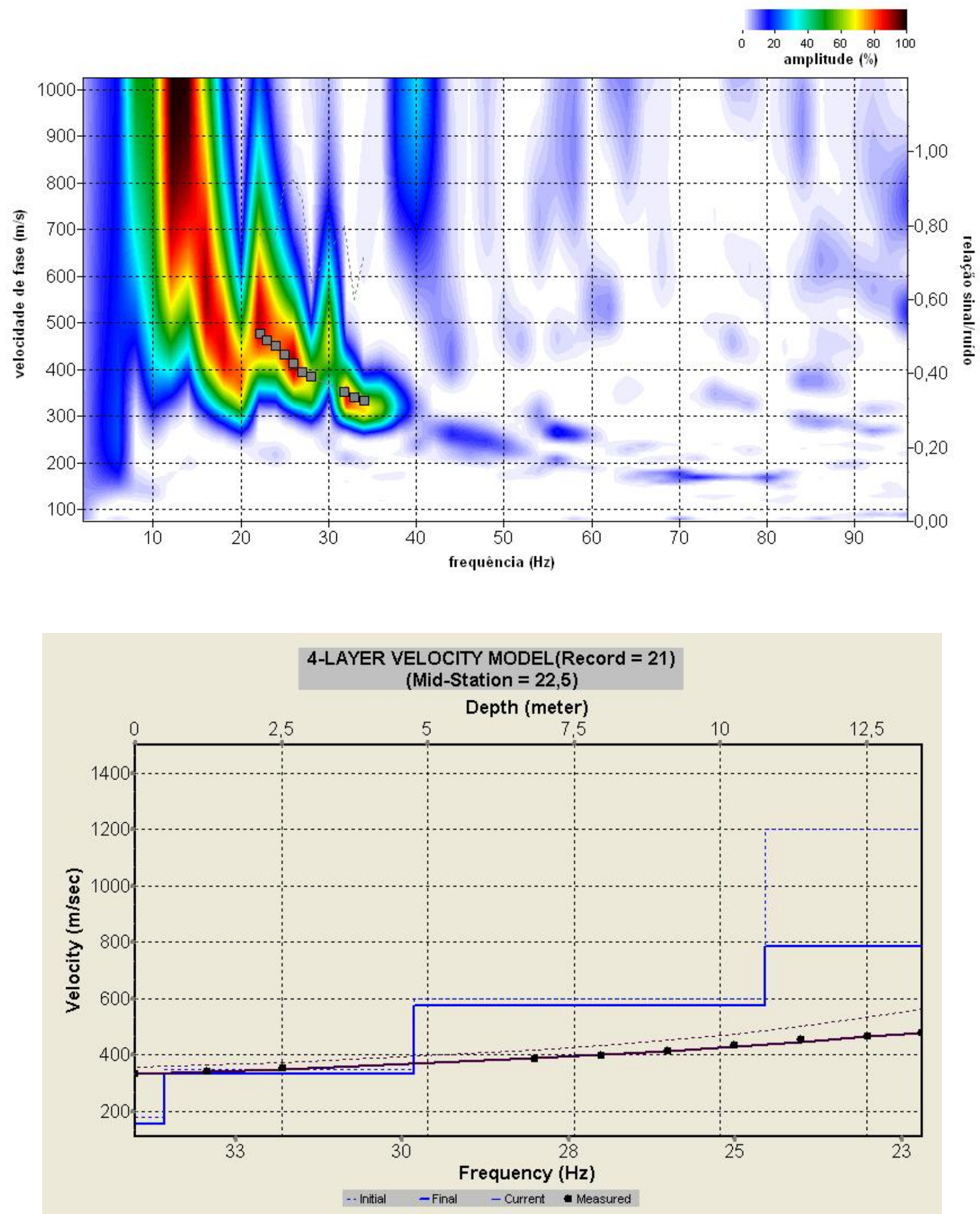

Figura 5 - Curva de dispersão e modelo final de inversão obtido dos sismogramas adquiridos com geofones de 4,5 Hz e afastamento mínimo de 10 metros. 